\title{
Spectroscopic study on deuterated benzenes. I. Microwave spectra and molecular structure in the ground state.
}

\section{$\operatorname{AUTHOR(S):~}$}

Kunishige, Sachi; Katori, Toshiharu; Baba, Masaaki; Nakajima, Masakazu; Endo, Yasuki

\section{CITATION:}

Kunishige, Sachi ... [et al]. Spectroscopic study on deuterated benzenes. I. Microwave spectra and molecular structure in the ground state.. The Journal of chemical physics 2015, 143(24): 244302.

\section{ISSUE DATE:}

2015-12-28

URL:

http://hdl.handle.net/2433/207681

\section{RIGHT:}

C 2015 AIP Publishing. This article may be downloaded for personal use only. Any other use requires prior permission of the author and AIP Publishing. The following article may be found at http://scitation.aip.org/content/aip/journal/jcp/143/24/10.1063/1.4937949. 


\section{AIP | $\begin{aligned} & \text { The Journal of } \\ & \text { chemical Physics }\end{aligned}$}

\section{Spectroscopic study on deuterated benzenes. I. Microwave spectra and molecular structure in the ground state}

Sachi Kunishige, Toshiharu Katori, Masaaki Baba, Masakazu Nakajima, and Yasuki Endo

Citation: The Journal of Chemical Physics 143, 244302 (2015); doi: 10.1063/1.4937949

View online: http://dx.doi.org/10.1063/1.4937949

View Table of Contents: http://scitation.aip.org/content/aip/journal/jcp/143/24?ver=pdfcov

Published by the AIP Publishing

\section{Articles you may be interested in}

On the molecular structure of $\mathrm{HOOO}$

J. Chem. Phys. 136, 034303 (2012); 10.1063/1.3673875

Single vibronic level emission spectroscopic studies of the ground state energy levels and molecular structures of jet-cooled $\mathrm{HGeBr}, \mathrm{DGeBr}, \mathrm{HGel}$, and DGel

J. Chem. Phys. 125, 114301 (2006); 10.1063/1.2355496

Determination of structural parameters for ferrocenecarboxaldehyde using Fourier transform microwave spectroscopy

J. Chem. Phys. 123, 054317 (2005); 10.1063/1.1993593

Fourier transform microwave spectroscopy of the $2 \Sigma+$ ground states of $\mathrm{YbX}(\mathrm{X}=\mathrm{F}, \mathrm{Cl}, \mathrm{Br})$ : Characterization of hyperfine effects and determination of the molecular geometries

J. Chem. Phys. 115, 6979 (2001); 10.1063/1.1404146

Microwave spectra, density functional theory calculations and molecular structure of acetylenemethyldioxorhenium

J. Chem. Phys. 113, 7891 (2000); 10.1063/1.1315611

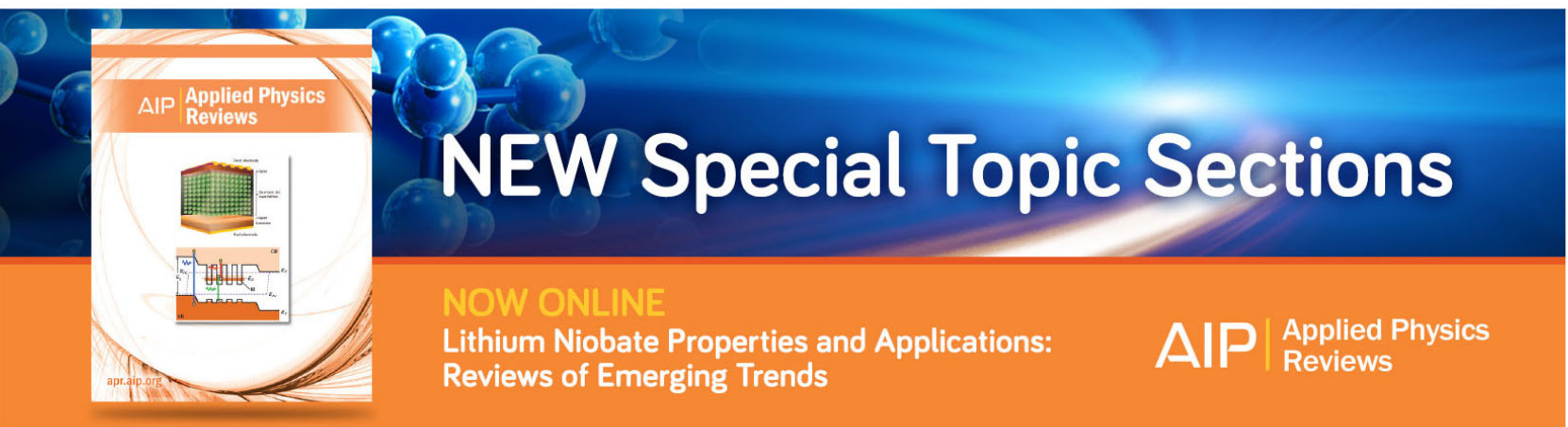




\title{
Spectroscopic study on deuterated benzenes. I. Microwave spectra and molecular structure in the ground state
}

\author{
Sachi Kunishige, ${ }^{1}$ Toshiharu Katori, ${ }^{1}$ Masaaki Baba,,${ }^{1, a)}$ Masakazu Nakajima, ${ }^{2}$ \\ and Yasuki Endo ${ }^{2}$ \\ ${ }^{1}$ Division of Chemistry, Graduate School of Science, Kyoto University, Kyoto 606-8502, Japan \\ ${ }^{2}$ Department of Basic Science, Graduate School of Arts and Sciences, The University of Tokyo, Komaba, \\ Meguro-ku, Tokyo 153-8902, Japan
}

(Received 14 September 2015; accepted 2 December 2015; published online 23 December 2015)

\begin{abstract}
We observed microwave absorption spectra of some deuterated benzenes and accurately determined the rotational constants of all H/D isotopomers in the ground vibrational state. Using synthetic analysis assuming that all bond angles are $120^{\circ}$, the mean bond lengths were obtained to be $r_{0}(\mathrm{C}-\mathrm{C})=1.3971 \AA$ and $r_{0}(\mathrm{C}-\mathrm{H})=r_{0}(\mathrm{C}-\mathrm{D})=1.0805 \AA$. It has been concluded that the effect of deuterium substitution on the molecular structure is negligibly small and that the mean bond lengths of $\mathrm{C}-\mathrm{H}$ and $\mathrm{C}-\mathrm{D}$ are identical unlike small aliphatic hydrocarbons, in which $r_{0}(\mathrm{C}-\mathrm{D})$ is about $5 \mathrm{m \AA}$ shorter than $r_{0}(\mathrm{C}-\mathrm{H})$. It is considered that anharmonicity is very small in the $\mathrm{C}-\mathrm{H}$ stretching vibration of aromatic hydrocarbons. (C) 2015 AIP Publishing LLC. [http://dx.doi.org/10.1063/1.4937949]
\end{abstract}

\section{INTRODUCTION}

Benzene is a key molecule that is of great importance investigating molecular structure and excited-state dynamics. We performed three spectroscopic experiments for jet-cooled deuterated benzenes, and analyzed the results based on theoretical considerations and $a b$ initio calculations. In Paper I, we present results on rotational constants of all the $H / D$ isotopomers which were obtained mainly by the analysis of microwave spectra. The main objective of this study is to accurately determine the molecular structure at the zerovibrational level in the electronic ground state. In Paper II, ${ }^{1}$ we present results of high-resolution laser spectroscopy for the $S_{1}{ }^{1} B_{2 u}-S_{0}{ }^{1} A_{1 g} 6_{0}^{1}\left(e_{2 g}\right)$ band and discuss the vibrational and rotational structure in the electronic excited state. In Paper III, ${ }^{2}$ we discuss the vibronic structure in the $S_{1}{ }^{1} B_{2 u}$ state and the radiationless transition in the electronic excited state, including "channel three," which would be observed as a drastic decrease in fluorescence quantum yield for high-vibrational levels. We also present the results of mass-selected resonance enhanced multiphoton ionization (REMPI) spectroscopy.

The structures of all the isotopomers of benzene including $\mathrm{H}$ and $\mathrm{D}$ atoms are illustrated in Fig. 1. There is only one species for $\mathrm{C}_{6} \mathrm{H}_{6}, \mathrm{C}_{6} \mathrm{H}_{5} \mathrm{D}, \mathrm{C}_{6} \mathrm{HD}_{5}$, and $\mathrm{C}_{6} \mathrm{D}_{6}$. For $\mathrm{C}_{6} \mathrm{H}_{4} \mathrm{D}_{2}$, there are three isotopomers: $o-\mathrm{C}_{6} \mathrm{H}_{4} \mathrm{D}_{2}, m-\mathrm{C}_{6} \mathrm{H}_{4} \mathrm{D}_{2}$, and $p-\mathrm{C}_{6} \mathrm{H}_{4} \mathrm{D}_{2}$, in which a pair of $\mathrm{D}$ atoms are substituted at the 1,2-(ortho), 1,3-(meta), and 1,4-(para) positions, respectively. For $\mathrm{C}_{6} \mathrm{H}_{2} \mathrm{D}_{4}$, there are three isomers structured in the reverse manner: $o-\mathrm{C}_{6} \mathrm{H}_{2} \mathrm{D}_{4}, m-\mathrm{C}_{6} \mathrm{H}_{2} \mathrm{D}_{4}$, and $p-\mathrm{C}_{6} \mathrm{H}_{2} \mathrm{D}_{4}$. Three isotopomers also exist for $\mathrm{C}_{6} \mathrm{H}_{3} \mathrm{D}_{3}$, which are designated $1,2,3-\mathrm{C}_{6} \mathrm{H}_{3} \mathrm{D}_{3}$, $1,2,4-\mathrm{C}_{6} \mathrm{H}_{3} \mathrm{D}_{3}$, and $1,3,5-\mathrm{C}_{6} \mathrm{H}_{3} \mathrm{D}_{3}$.

The benzene molecule is a planar regular hexagon $\left(D_{6 h}\right)$ and only two bond lengths, $r(\mathrm{C}-\mathrm{C})$ and $r(\mathrm{C}-\mathrm{H})$, are necessary

\footnotetext{
a) Author to whom correspondence should be addressed. Electronic mail: baba@kuchem.kyoto-u.ac.jp
}

to describe the structure. The molecular structure is possibly changed by deuterium substitution. For benzene, one is the change in the bond angles which distorts the regular hexagon. Another is the change in the bond lengths. We carefully discuss the difference between the $\mathrm{C}-\mathrm{H}$ and $\mathrm{C}-\mathrm{D}$ bond lengths. One can, however, only obtain one parameter from the rigid rotor rotational constants $A, B$, and $C$, because the relationship $A=B=2 C$ holds for the benzene molecules with $D_{6 h}$. A least-squares fit of rotational constants for deuterated molecules is a way to obtain more structural parameters. For $\mathrm{C}_{6} \mathrm{H}_{6}$ and $\mathrm{C}_{6} \mathrm{D}_{6}$, the rotational constants were determined precisely, ${ }^{3-7}$ and it was possible to evaluate two bond lengths assuming $r(\mathrm{C}-\mathrm{H})=r(\mathrm{C}-\mathrm{D}) .{ }^{6}$ The equilibrium bond length at the potential minimum is identical for different isotopes in the Born-Oppenheimer approximation, which is $r_{e}(\mathrm{C}-\mathrm{H})=r_{e}(\mathrm{C}-\mathrm{D})$.

However, the actual mean bond length $r_{0}(\mathrm{C}-\mathrm{D})$ at the zero-vibrational level is shorter than $r_{0}(\mathrm{C}-\mathrm{H})$ because of the anharmonicity in the potential energy. The increase in the mean bond length is smaller for the $\mathrm{C}-\mathrm{D}$ bond because the zero-point energy is smaller than that of the $\mathrm{C}-\mathrm{H}$ bond. The difference, $\Delta R=r_{0}(\mathrm{C}-\mathrm{H})-r_{0}(\mathrm{C}-\mathrm{D})$, has already been estimated for some small molecules. It was reported to be $5 \mathrm{m \AA}$ in $\mathrm{CH}^{8}{ }^{8} \mathrm{CH}_{3},{ }^{9,10}$ and $\mathrm{CH}_{4},{ }^{11}$ and $3-5 \mathrm{m \AA}$ in $\mathrm{CH}_{3} \mathrm{O}^{12}$ and $\mathrm{CH}_{3} \mathrm{~F}^{13} \Delta R$ is, however, suggested to be very small for large aromatic molecules such as benzene, ${ }^{7}$ naphthalene, ${ }^{7}$ and anthracene. ${ }^{14}$ To verify this point, we observed pure rotational transitions of jet-cooled deuterated benzenes using Fourier transform microwave (FTMW) spectroscopy. A small dipole moment arises from the zero-point vibration for isotopomers with low symmetry. The FTMW spectra of $\mathrm{C}_{6} \mathrm{H}_{5} \mathrm{D},{ }^{15} o$ $\mathrm{C}_{6} \mathrm{H}_{4} \mathrm{D}_{2}$, and $m-\mathrm{C}_{6} \mathrm{H}_{4} \mathrm{D}_{2}{ }^{16}$ have already been observed and the rotational constants were determined by Bauder's group. We have observed the FTMW spectra of $1,2,3-\mathrm{C}_{6} \mathrm{H}_{3} \mathrm{D}_{3}, o-\mathrm{C}_{6} \mathrm{H}_{2} \mathrm{D}_{4}$, $m-\mathrm{C}_{6} \mathrm{H}_{2} \mathrm{D}_{4}$, and $\mathrm{C}_{6} \mathrm{HD}_{5}$ and determined their rotational constants. In this article, we present the experimental results 

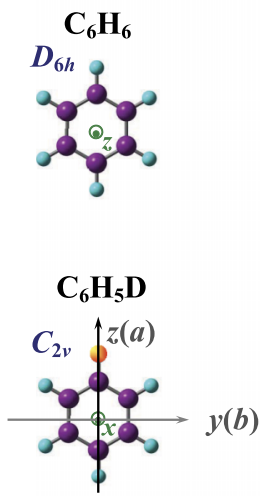

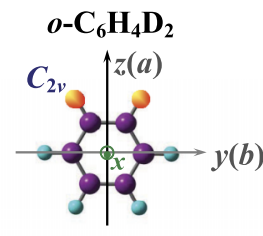

$m-\mathrm{C}_{6} \mathrm{H}_{4} \mathrm{D}_{2}$
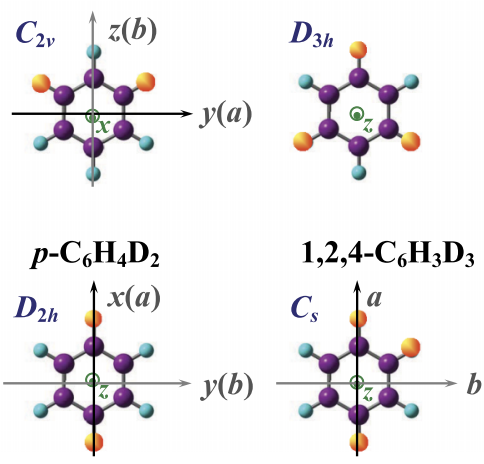

$1,3,5-C_{6} H_{3} D_{3}$

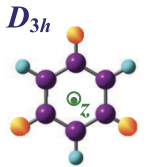

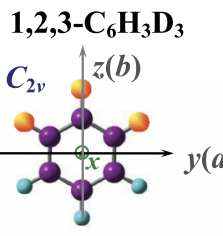

Fitructure of $\mathrm{H} / \mathrm{D}$ isotopomers of benzene.
$o-\mathbf{C}_{6} \mathbf{H}_{2} \mathbf{D}_{4}$

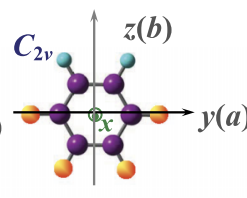

$m-\mathrm{C}_{6} \mathrm{H}_{2} \mathrm{D}_{4}$
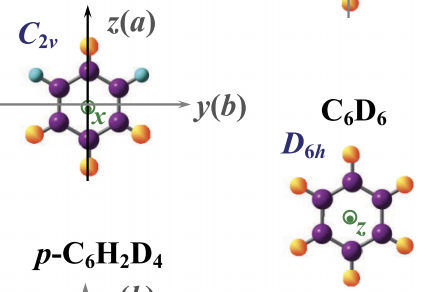

and discuss the molecular structure of benzene, particularly the mean bond lengths, $r_{0}(\mathrm{C}-\mathrm{H})$ and $r_{0}(\mathrm{C}-\mathrm{D})$.

\section{EXPERIMENTAL}

Deuterated benzenes were synthesized by maintaining the mixture of $\mathrm{C}_{6} \mathrm{H}_{6}$ and $\mathrm{C}_{6} \mathrm{D}_{6}$ at $800 \mathrm{~K}$ for $7 \mathrm{~h}$ with a small amount of aluminum powder. After the exchange reaction, the abundance ratio of isotopomers in the sample mixture became within the statistical limit distribution; as the number of $\mathrm{D}$ atoms increases, 1:6:15:20:15:6:1 for the mixture of $\mathrm{C}_{6} \mathrm{H}_{6}: \mathrm{C}_{6} \mathrm{D}_{6}=1: 1$. This $1: 1$ mixture was used in observing excitation spectra as shown in Papers II and III. ${ }^{1,2}$ In this microwave experiment, to increase the intensity, the mixture of $\mathrm{C}_{6} \mathrm{H}_{6}: \mathrm{C}_{6} \mathrm{D}_{6}=1: 3$ was used in which the abundance ratio was 1:8:25:40:35:16:3. The sample vapor (2\%) was mixed with the $\mathrm{Ne}$ gas and the mixed gas was expanded into a vacuum chamber from a pulsed nozzle to generate a supersonic jet.

A Balle-Flygare type FTMW spectrometer ${ }^{17}$ was used to observe pure rotational transitions. The microwave cavity was set coaxially to the jet. With this setup, a spectral line splits into two peaks by the Doppler effect (Fig. 2), and the transition frequency was calculated by taking the average. ${ }^{18}$

\section{RESULTS AND DISCUSSION}

We observed four or more rotational transitions for each isotopomer of $1,2,3-\mathrm{C}_{6} \mathrm{H}_{3} \mathrm{D}_{3}, o-\mathrm{C}_{6} \mathrm{H}_{2} \mathrm{D}_{4}, m-\mathrm{C}_{6} \mathrm{H}_{2} \mathrm{D}_{4}$, and $\mathrm{C}_{6} \mathrm{HD}_{5}$. Typical spectra are shown in Fig. 2, and all
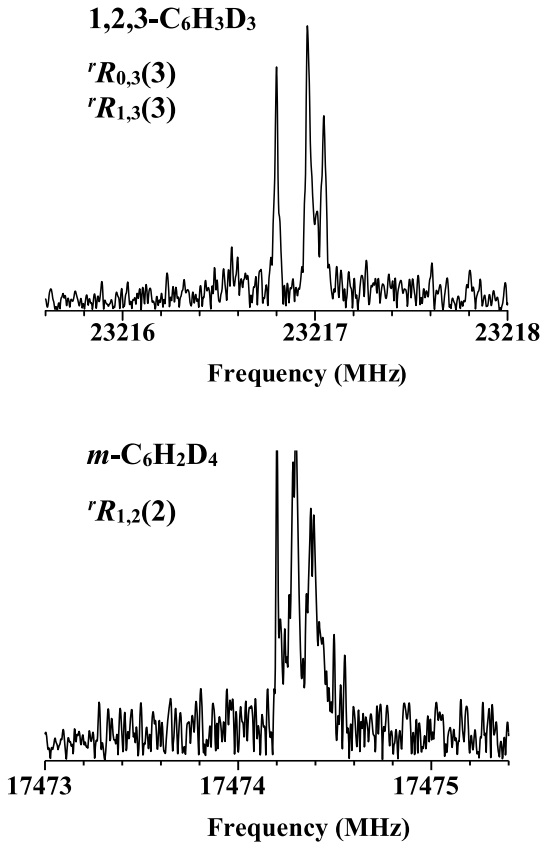
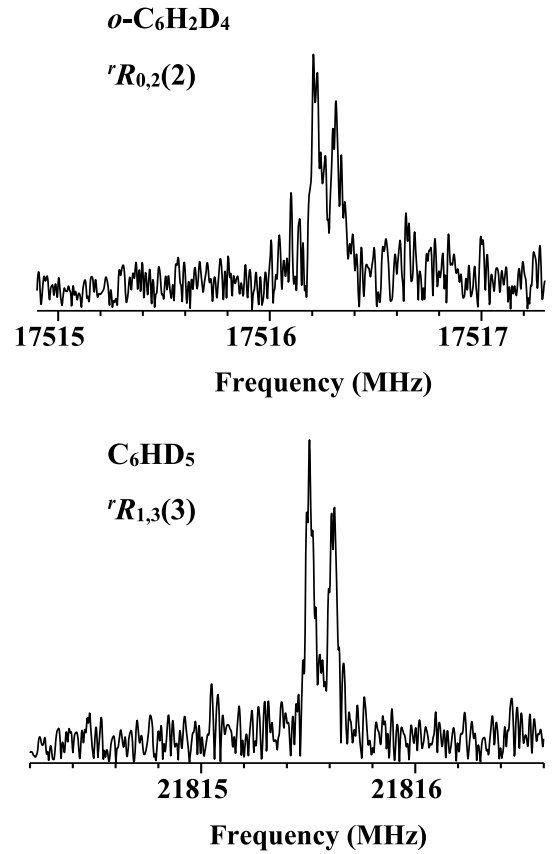

FIG. 2. Observed FTMW spectra of deuterated benzenes. A strong peak near the center is a noise caused by the strong microwave power. 
TABLE I. Frequencies and assignments of observed FTMW spectral lines.

\begin{tabular}{|c|c|c|c|c|c|c|c|c|}
\hline Molecule & Assignment & Frequency(MHz) & $J^{\prime}$ & $K_{a}^{\prime}$ & $K_{c}^{\prime}$ & $J^{\prime \prime}$ & $K_{a}^{\prime \prime}$ & $K_{c}^{\prime \prime}$ \\
\hline$o-\mathrm{C}_{6} \mathrm{H}_{4} \mathrm{D}_{2}$ & ${ }^{r} R_{1,3}(3)$ & $23962.921(7)$ & 4 & 1 & 4 & 3 & 1 & 3 \\
\hline \multirow[t]{6}{*}{$1,2,3-\mathrm{C}_{6} \mathrm{H}_{3} \mathrm{D}_{3}$} & ${ }^{r} R_{1,1}(1)$ & $12890.602(6)$ & 2 & 0 & 2 & 1 & 1 & 1 \\
\hline & ${ }^{r} R_{0,1}(1)$ & $12906.736(3)$ & 2 & 1 & 2 & 1 & 0 & 1 \\
\hline & ${ }^{r} R_{0,2}(2)$ & $18057.83(1)$ & 3 & 1 & 3 & 2 & 0 & 2 \\
\hline & ${ }^{r} R_{1,3}(3)$ & $23217.000(7)$ & 4 & 0 & 4 & 3 & 1 & 3 \\
\hline & ${ }^{r} R_{0,3}(3)$ & $23217.000(7)$ & 4 & 1 & 4 & 3 & 0 & 3 \\
\hline & ${ }^{r} R_{1,1}(2)$ & $23242.738(5)$ & 3 & 2 & 2 & 2 & 1 & 1 \\
\hline \multirow{6}{*}{$o-\mathrm{C}_{6} \mathrm{H}_{2} \mathrm{D}_{4}$} & ${ }^{r} R_{1,1}(1)$ & $12316.225(8)$ & 2 & 0 & 2 & 1 & 1 & 1 \\
\hline & ${ }^{r} R_{1,2}(2)$ & $17484.496(6)$ & 3 & 0 & 3 & 2 & 1 & 2 \\
\hline & ${ }^{r} R_{0,2}(2)$ & $17516.273(2)$ & 3 & 1 & 3 & 2 & 0 & 2 \\
\hline & ${ }^{r} R_{1,3}(3)$ & $22498.247(4)$ & 4 & 0 & 4 & 3 & 1 & 3 \\
\hline & ${ }^{r} R_{0,3}(3)$ & $22500.116(2)$ & 4 & 1 & 4 & 3 & 0 & 3 \\
\hline & ${ }^{r} R_{1,1}(2)$ & $22990.49(2)$ & 3 & 2 & 2 & 2 & 1 & 1 \\
\hline \multirow[t]{4}{*}{$m-\mathrm{C}_{6} \mathrm{H}_{2} \mathrm{D}_{4}$} & ${ }^{r} R_{1,2}(2)$ & $17474.339(3)$ & 3 & 1 & 3 & 2 & 1 & 2 \\
\hline & ${ }^{r} R_{0,2}(2)$ & $17499.975(8)$ & 3 & 0 & 3 & 2 & 0 & 2 \\
\hline & ${ }^{r} R_{1,3}(3)$ & $22481.543(2)$ & 4 & 1 & 4 & 3 & 1 & 3 \\
\hline & ${ }^{r} R_{0,3}(3)$ & $22483.007(3)$ & 4 & 0 & 4 & 3 & 0 & 3 \\
\hline \multirow[t]{4}{*}{$\mathrm{C}_{6} \mathrm{HD}_{5}$} & ${ }^{r} R_{1,1}(1)$ & $11953.075(5)$ & 2 & 0 & 2 & 1 & 1 & 1 \\
\hline & ${ }^{r} R_{0,1}(1)$ & $12270.076(7)$ & 2 & 1 & 2 & 1 & 0 & 1 \\
\hline & ${ }^{r} R_{1,3}(3)$ & $21815.561(2)$ & 4 & 0 & 4 & 3 & 1 & 3 \\
\hline & ${ }^{r} R_{0,3}(3)$ & 218 17.101(4) & 4 & 1 & 4 & 3 & 0 & 3 \\
\hline
\end{tabular}

the transition frequencies are presented in Table I. Because similar experiments were performed previously for $\mathrm{C}_{6} \mathrm{H}_{5} \mathrm{D}$, $o$ - $\mathrm{C}_{6} \mathrm{H}_{4} \mathrm{D}_{2}$, and $m-\mathrm{C}_{6} \mathrm{H}_{4} \mathrm{D}_{2},{ }^{15,16}$ pure rotational transitions have been observed for all the microwave-active deuterated benzenes except $1,2,4-\mathrm{C}_{6} \mathrm{H}_{3} \mathrm{D}_{3}$. It was difficult to accurately determine the higher-order rotational constants because of the limited number of observed transitions. However, the least-square fit was not good enough without the centrifugal distortion terms. We, therefore, fixed them to the averaged values of $\mathrm{C}_{6} \mathrm{H}_{6}$ and $\mathrm{C}_{6} \mathrm{D}_{6}$ for $D_{J}, D_{J K}$, and $D_{K}$, and then obtained reliable values of the rigid-rotor rotational constants $A, B$, and $C$. The best-fit results are listed in Table II, (b). For $o-\mathrm{C}_{6} \mathrm{H}_{4} \mathrm{D}_{2}$, we obtained slightly different values from the reported ones by Oldani et al., ${ }^{16}$ because a new transition was incorporated in a least squares fit. Oldani et al. fitted the centrifugal distortion constants of $o-\mathrm{C}_{6} \mathrm{H}_{4} \mathrm{D}_{2}$ separately, and their rotational constants are considered to be more accurate than ours. However, we used our values here because they improved consistency in a synthetic analysis for all isotopomers. Actually, centrifugal distortion constants did not affect the rigid-rotor rotational constants $A, B$, and $C$ within the experimental error. For $\mathrm{C}_{6} \mathrm{H}_{6}$ and $\mathrm{C}_{6} \mathrm{D}_{6}$, we used the values that were previously determined by ultrahigh-resolution laser spectroscopy. ${ }^{5,6}$

The $A, B$, and $C$ values in Table II, (a) are the calculated rotational constants of all H/D isotopomers, which were

TABLE II. Rotational constants $\left(\mathrm{cm}^{-1}\right)$ of all benzene H/D isotopomers at the zero-vibrational levels in the $S_{0}$ state.

\begin{tabular}{lccccccc}
\hline \hline & \multicolumn{3}{c}{ (a) By $R_{0}$ Structure } & & \multicolumn{3}{c}{ (b) By Observation } \\
\cline { 2 - 3 } \cline { 7 - 8 } & $A\left(\mathrm{~cm}^{-1}\right)$ & $B\left(\mathrm{~cm}^{-1}\right)$ & $C\left(\mathrm{~cm}^{-1}\right)$ & & $A\left(\mathrm{~cm}^{-1}\right)$ & $B\left(\mathrm{~cm}^{-1}\right)$ & $C\left(\mathrm{~cm}^{-1}\right)$ \\
\hline $\mathrm{C}_{6} \mathrm{H}_{6}$ & 0.18977 & 0.18977 & 0.09488 & & $0.1897717(3)$ & $0.1897717(3)$ & $0.0948859(2)$ \\
$\mathrm{C}_{6} \mathrm{H}_{5} \mathrm{D}$ & 0.18977 & 0.17758 & 0.09173 & & $0.1897694(2)$ & $0.1775873(2)$ & $0.0917193(2)$ \\
$o-\mathrm{C}_{6} \mathrm{H}_{4} \mathrm{D}_{2}$ & 0.18339 & 0.17225 & 0.08882 & & $0.1833946(3)$ & $0.1722596(3)$ & $0.0888103(2)$ \\
$m-\mathrm{C}_{6} \mathrm{H}_{4} \mathrm{D}_{2}$ & 0.18355 & 0.17184 & 0.08875 & & $0.1835492(3)$ & $0.1718540(3)$ & $0.0887398(3)$ \\
$p-\mathrm{C}_{6} \mathrm{H}_{4} \mathrm{D}_{2}$ & 0.18977 & 0.16660 & 0.08872 & & & \\
$1,3,5-\mathrm{C}_{6} \mathrm{H}_{3} \mathrm{D}_{3}$ & 0.17184 & 0.17184 & 0.08592 & & & \\
$1,2,3-\mathrm{C}_{6} \mathrm{H}_{3} \mathrm{D}_{3}$ & 0.17238 & 0.17184 & 0.08606 & & $0.1723865(5)$ & $0.171850(2)$ & $0.0860455(2)$ \\
$1,2,4-\mathrm{C}_{6} \mathrm{H}_{3} \mathrm{D}_{3}$ & 0.18183 & 0.16302 & 0.08596 & & & \\
$o-\mathrm{C}_{6} \mathrm{H}_{2} \mathrm{D}_{4}$ & 0.17224 & 0.16166 & 0.08339 & & $0.1722430(2)$ & $0.1616723(2)$ & $0.0833841(1)$ \\
$m-\mathrm{C}_{6} \mathrm{H}_{2} \mathrm{D}_{4}$ & 0.17184 & 0.16178 & 0.08333 & & $0.171852(4)$ & $0.161789(4)$ & $0.083321(1)$ \\
$p-\mathrm{C}_{6} \mathrm{H}_{2} \mathrm{D}_{4}$ & 0.17743 & 0.15701 & 0.08330 & & & \\
$\mathrm{C}_{6} \mathrm{HD}_{5}$ & 0.16672 & 0.15701 & 0.08086 & & $0.166721(5)$ & $0.157016(5)$ & $0.080855(2)$ \\
$\mathrm{C}_{6} \mathrm{D}_{6}$ & 0.15701 & 0.15701 & 0.07850 & $0.1570128(2)$ & $0.1570128(2)$ & $0.0785064(1)$ \\
\hline \hline
\end{tabular}


obtained by assuming the common $R_{0}$ structure, as described below. We also used the relationship between the $A, B$, and $C$ values derived by structural considerations. For example, the $B$ value of $\mathrm{C}_{6} \mathrm{HD}_{5}$ must be identical to the $A$ and $B$ values of $\mathrm{C}_{6} \mathrm{D}_{6}$, and the $B$ value of $m-\mathrm{C}_{6} \mathrm{H}_{4} \mathrm{D}_{2}$ must be identical to the $B$ value of $1,3,5-\mathrm{C}_{6} \mathrm{H}_{3} \mathrm{D}_{3}$. The resultant values are in agreement with those obtained by the least-squares fit of each isotopomer, with a standard deviation of $1 \times 10^{-5} \mathrm{~cm}^{-1}$.

We discuss how to accurately determine the geometrical structure of benzene from the rigid rotor rotational constants of deuterated isotopomers, especially the $\mathrm{C}-\mathrm{H}$ bond length. Several models have been proposed to represent the molecular structure, such as $r_{e}, r_{0}, r_{g}, r_{s}, r_{m}$, and $r$ structures. The $r_{e}$ structure is the equilibrium structure where nuclei are frozen at the potential minimum, which is identical for different isotopomers in the Born-Oppenheimer approximation. An actual molecule takes a slightly different structure because of the zero-point vibration. The mean displacements of normal coordinates are not zero, including those of a $\mathrm{C}-\mathrm{H}$ stretching mode: $\langle\Delta Q\rangle \neq 0 .{ }^{19}$

The mean atomic positions are different from the equilibrium positions, which are represented by the $r_{0}$ structure. The rotational constant is approximately given by

$$
\begin{aligned}
B_{0} & =B_{e}-\frac{1}{2} \sum_{i} \alpha_{i} \\
& =B_{e}-\frac{1}{2} \sum_{i}\left(\alpha_{i}^{\text {harm }}+\alpha_{i}^{\text {anharm }}\right),
\end{aligned}
$$

where $\alpha_{i}$ is the anharmonic constant of the $i$ th normal mode. $\alpha_{i}^{\text {harm }}$ represents anharmonicity which exists in the harmonic potential. Because it is difficult to determine all the $\alpha_{i}$ values for a large molecule, it is difficult to obtain the $r_{e}$ structure. Another experimental method to accurately determine the geometrical structure is electron diffraction for gas phase molecules. ${ }^{20}$ The mean distances between the identical atoms are calculated from the diffraction angles, which lead to the $r_{g}$ structure. The mean bond length is given by

$$
r_{g}=r_{e}+\langle\mathrm{CRD}\rangle+\langle\Delta z\rangle+\frac{\left\langle\Delta x^{2}\right\rangle+\left\langle\Delta y^{2}\right\rangle}{2 r_{e}}+\cdots,
$$

where $\langle\mathrm{CRD}\rangle$ is the centrifugal distortion term. $\langle\Delta z\rangle$ is the mean displacement of stretching along the bond axis, and $\left\langle\Delta x^{2}\right\rangle$ and $\left\langle\Delta y^{2}\right\rangle$ are the perpendicular components of the mean square displacement. $\left\langle\Delta x^{2}\right\rangle$ and $\left\langle\Delta y^{2}\right\rangle$ include only the harmonic terms, whereas $\langle\Delta z\rangle$ arises from the anharmonic effect. The moment of inertia in the zero-vibrational level can be extracted from Eqs. (2) and (3), and we obtain

$$
\begin{aligned}
I_{z} & =I_{0}\left(1-\sum_{i} \frac{\alpha_{i}^{\text {harm }}}{2 B_{0}}\right) \\
& =I_{e}\left(1+\sum_{i} \frac{\alpha_{i}^{\text {anharm }}}{2 B_{0}}\right) .
\end{aligned}
$$

Although this is a favorable way to determine the bond length, the experimental accuracy is not higher than high-resolution spectroscopy. The results of ethylene- $h_{4}$ and ethylene- $d_{4}$ are not reliable. ${ }^{21,22}$
We then considered the $r_{s}$ structure, which is obtained from the difference of moments of inertia between the isotopomers, $\Delta I$, assuming that the position of nuclei is not changed by isotopic substitution. ${ }^{19,23}$ We started from this model to analyze the rotational constants of deuterated benzenes. However, we must take into account the change in the $\mathrm{C}-\mathrm{H}$ bond length, while the $\mathrm{C}-\mathrm{C}$ bond length and bond angles are assumed to be identical for all the isotopomers. We define a fitting parameter

$$
\Delta R=r_{0}(\mathrm{C}-\mathrm{H})-r_{0}(\mathrm{C}-\mathrm{D})
$$

and search the best-fit value that minimizes the mean-square deviation of the observed rotational constants. We call this the $R_{0}$ structure. The origin is set to the center of the regular hexagon composed of $\mathrm{C}$ atoms. In the intramolecular coordinates of each atomic nucleus $\boldsymbol{r}_{i}\left(x_{i}, y_{i}, z_{i}\right), \boldsymbol{r}_{0}$ is given by the moment of inertia and is represented by a tensor whose elements are obtained by ${ }^{19}$

$$
\begin{aligned}
& I_{x x}=\sum_{i} m_{i}\left(y_{i}^{2}+z_{i}^{2}\right)-\frac{\left(\sum_{i} m_{i} y_{i}\right)^{2}}{\sum_{i} m_{i}}-\frac{\left(\sum_{i} m_{i} z_{i}\right)^{2}}{\sum_{i} m_{i}}, \\
& I_{x y}=-\sum_{i} m_{i} x_{i} y_{i}+\frac{\left(\sum_{i} m_{i} x_{i}\right)\left(\sum_{i} m_{i} y_{i}\right)}{\sum_{i} m_{i}}-\frac{\left(\sum_{i} m_{i} z_{i}\right)^{2}}{\sum_{i} m_{i}} .
\end{aligned}
$$

The $\mathrm{C}_{6} \mathrm{H}_{6}$ structure with the $D_{6 h}$ symmetry is assumed to remain in the $r_{s}$ structure of all deuterated isotopomers. There are differences in the moment of inertia around the $\alpha$ axis $(\alpha=x, y, z)$ between $\mathrm{C}_{6} \mathrm{H}_{6}$ and a deuterium-substituted molecule (the molecular weight, $M^{\prime}$ ), and the following relationships are derived from Eqs. (7) and (8):

$$
\begin{aligned}
\Delta I_{\beta \beta} & =\frac{f_{\beta} M^{\prime}-g^{2} \Delta m}{M^{\prime}} \cdot \Delta m \cdot r_{s}(\mathrm{H})^{2}, \\
\Delta I_{z z} & =f_{z} \cdot \Delta m \cdot r_{s}(\mathrm{H})^{2}, \\
\Delta I_{\alpha \beta} & =0 .
\end{aligned}
$$

Here, $\Delta m$ is the difference between the atomic masses, $\Delta m=m_{\mathrm{D}}-m_{\mathrm{H}}$, and $\beta$ is either $x$ or $y . f_{\beta}, f_{z}$, and $g$ are the parameters that depend on the isotopomer and molecular axis as indicated in Table III. The $z$ axis is in the direction of the symmetry axis. $r_{s}(\mathrm{H})$ is the distance from the origin to the $\mathrm{H}$ atom and it is given by

$$
r_{s}(\mathrm{H})=r_{s}(\mathrm{C}-\mathrm{H})+r_{s}(\mathrm{C}-\mathrm{C}) .
$$

TABLE III. Coefficients $f_{x}, f_{y}, f_{z}$, and $g$ for benzene H/D isotopomers.

\begin{tabular}{lccccccccc}
\hline \hline & \multicolumn{4}{c}{ From $\mathrm{C}_{6} \mathrm{H}_{6}$} & & \multicolumn{5}{c}{ From $\mathrm{C}_{6} \mathrm{D}_{6}$} \\
\cline { 2 - 4 } \cline { 7 - 9 } Molecule & $f_{x}$ & $f_{y}$ & $f_{z}$ & $g$ & & $f_{x}$ & $f_{y}$ & $f_{z}$ & $g$ \\
\hline $\mathrm{C}_{6} \mathrm{H}_{6}$ & & & & & -6 & -3 & -3 & 0 \\
$\mathrm{C}_{6} \mathrm{H}_{5} \mathrm{D}$ & 1 & 1 & 0 & 1 & -5 & -2 & -3 & 1 \\
$o-\mathrm{C}_{6} \mathrm{H}_{4} \mathrm{D}_{2}$ & 2 & $3 / 2$ & $1 / 2$ & $\sqrt{3}$ & -4 & $-3 / 2$ & $-5 / 2$ & $\sqrt{3}$ \\
$m-\mathrm{C}_{6} \mathrm{H}_{4} \mathrm{D}_{2}$ & 2 & $1 / 2$ & $3 / 2$ & 1 & -4 & $-5 / 2$ & $-3 / 2$ & 1 \\
$p-\mathrm{C}_{6} \mathrm{H}_{4} \mathrm{D}_{2}$ & 3 & $3 / 2$ & $3 / 2$ & 2 & -3 & $-3 / 2$ & $-3 / 2$ & 2 \\
$o-\mathrm{C}_{6} \mathrm{H}_{2} \mathrm{D}_{4}$ & 4 & $3 / 2$ & $5 / 2$ & $\sqrt{3}$ & -2 & $-3 / 2$ & $-1 / 2$ & $\sqrt{3}$ \\
$m-\mathrm{C}_{6} \mathrm{H}_{2} \mathrm{D}_{4}$ & 4 & $5 / 2$ & $3 / 2$ & 1 & -2 & $-1 / 2$ & $-3 / 2$ & 1 \\
$\mathrm{C}_{6} \mathrm{HD}_{5}$ & 5 & 2 & 3 & 1 & -1 & -1 & 0 & 1 \\
$\mathrm{C}_{6} \mathrm{D}_{6}$ & 6 & 3 & 3 & 0 & & & & \\
\hline \hline
\end{tabular}


To search the $R_{0}$ structure and introduce $\Delta R$, it is useful to calculate the change in the moment of inertia by substituting the $R_{0}$ structure in Eq. (7). If we assume that $(\Delta R)^{2}$ is much smaller than $R_{0}(\mathrm{H})$, the elements of the $\Delta I$ tensor are expressed as

$$
\begin{aligned}
\Delta I_{\beta \beta}= & f_{\beta}\left[m_{\mathrm{D}}\left\{R_{0}(\mathrm{H})-\Delta R\right\}^{2}-m_{\mathrm{H}} R_{0}(\mathrm{H})^{2}\right] \\
& -\frac{g^{2}}{M^{\prime}}\left[m_{\mathrm{D}}\left\{R_{0}(\mathrm{H})-\Delta R\right\}-m_{\mathrm{H}} R_{0}(\mathrm{H})\right]^{2} \\
= & \frac{f_{\beta} M^{\prime}-g^{2} \Delta m}{M^{\prime}}\left\{\Delta m R_{0}(\mathrm{H})^{2}-2 m_{\mathrm{D}} \Delta R \cdot R_{0}(\mathrm{H})\right\} \\
& +\frac{m_{\mathrm{D}}}{M^{\prime}}\left(f_{\beta} M^{\prime}-g^{2} m_{\mathrm{D}}\right)(\Delta R)^{2} \\
\simeq & \frac{f_{\beta} M^{\prime}-g^{2} \Delta m}{M^{\prime}}\left\{\Delta m R_{0}(\mathrm{H})^{2}-2 m_{\mathrm{D}} R_{0}(\mathrm{H}) \cdot \Delta R\right\}, \\
\Delta I_{z z}= & f_{z}\left\{\Delta m R_{0}(\mathrm{H})^{2}-2 m_{\mathrm{D}} R_{0}(\mathrm{H}) \cdot \Delta R+m_{\mathrm{D}}(\Delta R)^{2}\right\} \\
\simeq & f_{z}\left\{\Delta m R_{0}(\mathrm{H})^{2}-2 m_{\mathrm{D}} R_{0}(\mathrm{H}) \cdot \Delta R\right\} .
\end{aligned}
$$

By comparing these equations with Eqs. (9) and (10), we obtain

$$
r_{s}(\mathrm{H})^{2}=R_{0}(\mathrm{H})^{2}-\frac{2 m_{\mathrm{D}}}{\Delta m} R_{0}(\mathrm{H}) \cdot \Delta R .
$$

Therefore, the $r_{s}$ bond length is approximately given by

$$
r_{s}(\mathrm{H})=R_{0}(\mathrm{H})-\frac{m_{\mathrm{D}}}{\Delta m} \Delta R .
$$

$I_{y y}$ which is the moment of inertia along the in-plane axis perpendicular to the symmetry axis can be derived by starting the deuterium substitution from $\mathrm{C}_{6} \mathrm{H}_{6}$,

$$
\begin{aligned}
I_{y y}\left(\mathrm{C}_{6} \mathrm{H}_{6}\right) & =3 m_{\mathrm{C}} R_{0}(\mathrm{C})^{2}+3 m_{\mathrm{H}} R_{0}(\mathrm{H})^{2} \\
& =3 m_{\mathrm{C}} R_{0}(\mathrm{C})^{2}+3 m_{\mathrm{H}}\left[r_{s}(\mathrm{H})^{2}+\frac{2 m_{\mathrm{D}}}{\Delta m} r_{s}(\mathrm{H}) \cdot \Delta R\right] .
\end{aligned}
$$

Conversely, the hydrogen substitution from $\mathrm{C}_{6} \mathrm{D}_{6}$ leads to

$$
\begin{aligned}
I_{y y}\left(\mathrm{C}_{6} \mathrm{D}_{6}\right) & =3 m_{\mathrm{C}} R_{0}(\mathrm{C})^{2}+3 m_{\mathrm{D}}\left(R_{0}(\mathrm{H})-\Delta R\right)^{2} \\
& =3 m_{\mathrm{C}} R_{0}(\mathrm{C})^{2}+3 m_{\mathrm{D}}\left[r_{s}(\mathrm{H})^{2}+\frac{2 m_{\mathrm{H}}}{\Delta m} r_{s}(\mathrm{H}) \cdot \Delta R\right] .
\end{aligned}
$$

Because $I_{y y}\left(\mathrm{C}_{6} \mathrm{H}_{6}\right)$ and $I_{y y}\left(\mathrm{C}_{6} \mathrm{D}_{6}\right)$ were accurately determined by ultrahigh-resolution spectroscopy, it is possible to determine the best-fit value of $\Delta R . R_{0}(\mathrm{C})$ can be determined from $r_{s}(\mathrm{H})$ and $\Delta R$ using Eqs. (21) and (23). $R_{0}(\mathrm{H})$ can be determined in the same way using Eq. (19). The following square deviation parameter was used as the objective criteria of evaluation:

$\sigma^{2}=\sum\left[\left\{I_{a a}(\text { calc })-I_{a a}(\mathrm{obs})\right\}^{2}+\left\{I_{b b}(\text { calc })-I_{b b}(\mathrm{obs})\right\}^{2}\right]$.

We searched for the best $R_{0}$ structure of deuterated benzenes, using $R_{0}(\mathrm{C}-\mathrm{C}), R_{0}(\mathrm{C}-\mathrm{H})$, and $\Delta R$, which minimized the value of this error parameter $\sigma^{2}$. The results with respect to $R_{0}(\mathrm{C})$ are shown in Fig. 3. Because two $R_{0}(\mathrm{C})$ values were obtained from Eqs. (21) and (23), we used the averaged values for the least-squares fit, which is expressed as $R_{0}^{A}(\mathrm{C})$. We assume this

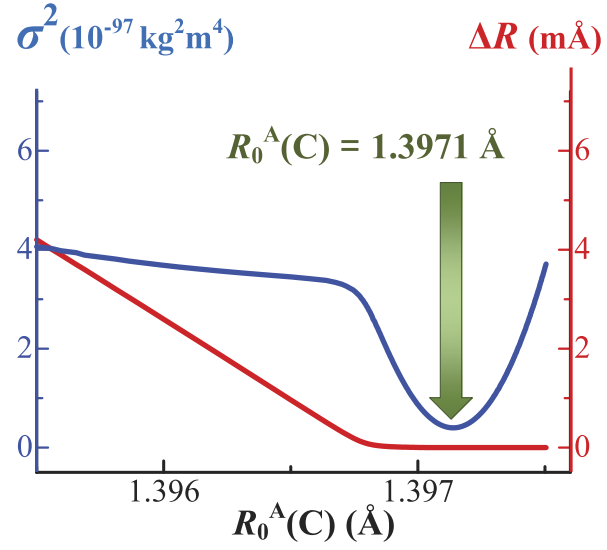

FIG. 3. Change in $\sigma^{2}$ and $\Delta R$ with respect to the $\mathrm{C}-\mathrm{C}$ mean bond length $R_{0}^{A}(\mathrm{C})$.

$R_{0}^{A}(\mathrm{C})$ to be $R_{0}(\mathrm{C}-\mathrm{C}) . \sigma^{2}$ showed the minimum at $R_{0}(\mathrm{C}-\mathrm{C})$ $=1.3971 \AA$ and $R_{0}(\mathrm{C}-\mathrm{H})=1.0805 \AA$, with $\Delta R=0.0 \mathrm{~m} \AA . \Delta R$ was zero in the range of $R_{0}(\mathrm{C}-\mathrm{H})<1.0821 \AA$. We therefore used these value as the best-fit $R_{0}$ structure. The standard deviation error was estimated to be $\pm 0.3 \mathrm{~mA}$ for $R_{0}(\mathrm{C}-\mathrm{H})$ by comparing the determined rotational constants and the calculated values using this final structure. In Table IV, this $R_{0}$ structure is compared with the previously reported structures that were obtained assuming that $r_{0}(\mathrm{C}-\mathrm{H})=r_{0}(\mathrm{C}-\mathrm{D})$ in addition to the results of $a b$ initio calculations. ${ }^{7}$ All values are in agreement within the error of $1 \mathrm{~m} \AA$ for $R_{0}(\mathrm{C}-\mathrm{H})$ and $0.2 \mathrm{~m} \AA$ for $R_{0}(\mathrm{C}-\mathrm{C})$. Thus, the mean bond length is approximately the same for $\mathrm{C}-\mathrm{H}$ and $\mathrm{C}-\mathrm{D}$ in the benzene molecule.

Finally, we consider the $r_{e}$ structure. Pliva et al. ${ }^{3}$ proposed a modified model, $r$ structure, where the equilibrium rotational constants of an isotopically substituted molecule were derived from the mean rotational constants and a molecular weight of $\mathrm{C}_{6} \mathrm{H}_{6}$ using an approximate relationship for a symmetric top molecule,

$$
B_{0}^{\prime}=B_{e}^{\prime}-1.787 \times 10^{-3}\left(\frac{B_{0}^{\prime}}{B_{0}}\right)^{2}\left(\frac{M_{0}^{\prime}}{M_{0}}\right)^{1 / 2} .
$$

Assuming that Eq. (25) holds for an asymmetric top molecule, we obtained the $r_{e}$ structure from the rotational constants of deuterated benzenes. The results are shown in Table V and compared with other experimental results and $a b$ initio calculations. It should be noted, however, that $R_{e}(\mathrm{C}-\mathrm{H})$ is not shorter than $R_{0}(\mathrm{C}-\mathrm{H})$ in the analysis, using the method of Pliva et al. This $r_{e}$ structure is thus not yet accurate. High level calculations for the vibration-rotation constants should be performed to accurately determine the $r_{e}$ structure of benzene.

TABLE IV. $r_{0}$ structure of benzene ( $\AA$ ).

\begin{tabular}{lcc}
\hline \hline & $r_{0}(\mathrm{C}-\mathrm{H})=r_{0}(\mathrm{C}-\mathrm{D})$ & $R_{0}(\mathrm{C}-\mathrm{C})$ \\
\hline This work & 1.0805 & 1.3971 \\
Doi et al. $^{6}$ & 1.0807 & 1.3971 \\
Pliva et al. $^{3}$ & 1.0815 & 1.3969 \\
Baba et al. $^{7}$ & 1.0817 & 1.3969 \\
\hline \hline
\end{tabular}


TABLE V. $r_{e}$ structure of benzene $(\AA)$.

\begin{tabular}{lcc}
\hline \hline & $R_{e}(\mathrm{C}-\mathrm{H})=R_{e}(\mathrm{C}-\mathrm{D})$ & $R_{e}(\mathrm{C}-\mathrm{C})$ \\
\hline This work & 1.0864 & 1.3892 \\
Pliva et al. ${ }^{3}$ & 1.0857 & 1.3893 \\
${\text { Gauss } \text { et al. }(\text { ab } \text { initio })^{24}}^{4}$ & 1.0800 & 1.3911 \\
\hline
\end{tabular}

It should be noted that the anharmonic effect on the $\mathrm{C}-\mathrm{H}$ bond is suggested to be small, suggesting that $R_{0}(\mathrm{C}-\mathrm{D})$ is approximately identical to $R_{0}(\mathrm{C}-\mathrm{H})$. As a result, our $R_{0}$ structure is the same as the $r_{0}$ structure in $D_{6 h}$ symmetry. The values of inertial defect, which is attributed to mainly out-of-plane zero-point vibrations, ${ }^{25,26}$ are consistent with this result. These effects on the rotational constants are estimated to be less than $0.03 \%,{ }^{4,5}$ and the obtained $R_{0}$ structure is substantially identical to the $r_{0}$ structure. This means that the mean structure of benzene is a planar regular hexagon with $r_{0}(\mathrm{C}-\mathrm{H})=r_{0}(\mathrm{C}-\mathrm{D})$.

\section{CONCLUSIONS}

The rotational constants for several deuterated benzenes at the zero-vibrational levels in the ground state were determined using FTMW spectroscopy. We performed a synthetic best-fit analysis to determine the mean bond lengths, and finally obtained $r_{0}(\mathrm{C}-\mathrm{C})=1.3971 \AA$ and $r_{0}(\mathrm{C}-\mathrm{H})=1.0805 \AA$. We also found that $r_{0}(\mathrm{C}-\mathrm{H})=r_{0}(\mathrm{C}-\mathrm{D})$. This relationship was suggested to be valid also in naphthalene and anthracene. We consider that the negligibly small deuterium effect on the $\mathrm{C}-\mathrm{H}$ mean bond length is common for aromatic hydrocarbons. It may be attributed to a mixing of the normal modes, which makes the anharmonicity small for the $\mathrm{C}-\mathrm{H}$ stretching.

\section{ACKNOWLEDGMENTS}

The authors thank Dr. Kazuyoshi Kanamori and Professor Kazuki Nakanishi (Division of Chemistry, Graduate School of Science, Kyoto University) for their help to synthesize mixed sample of deuterated benzenes.

${ }^{1}$ S. Kunishige, T. Katori, M. Baba, M. Hayashi, H. Hasegawa, and Y. Ohshima, J. Chem. Phys. 143, 244303 (2015).

${ }^{2}$ S. Kunishige, T. Katori, M. Kawabata, T. Yamanaka, and M. Baba, J. Chem. Phys. 143, 244304 (2015).

${ }^{3}$ J. Pliva, J. W. C. Johns, and L. Goodman, J. Mol. Spectrosc. 140, 214 (1990). ${ }^{4}$ M. Okruss, R. Müller, and A. Hese, J. Mol. Spectrosc. 193, 293 (1999).

${ }^{5}$ A. Doi, S. Kasahara, H. Katô, and M. Baba, J. Chem. Phys. 120, 6439 (2004).

${ }^{6}$ A. Doi, M. Baba, S. Kasahara, and H. Katô, J. Mol. Spectrosc. 227, 180 (2004).

${ }^{7}$ M. Baba, Y. Kowaka, U. Nagashima, T. Ishimoto, H. Goto, and N. Nakayama, J. Chem. Phys. 135, 054305 (2011).

${ }^{8}$ D. R. Herschbach and V. W. Laurie, J. Chem. Phys. 37, 1668 (1962).

${ }^{9}$ E. Hirota and C. Yamada, J. Mol. Spectrosc. 96, 175 (1982).

${ }^{10}$ T. J. Sears, J. M. Flye, V. Spirko, and W. P. Kraemer, J. Chem. Phys. 90, 2125 (1989).

${ }^{11}$ E. Hirota, J. Mol. Spectrosc. 77, 213 (1979).

${ }^{12}$ J. Liu, M.-W. Chen, D. Melnik, T. A. Miller, Y. Endo, and E. Hirota, J. Chem. Phys. 130, 074303 (2009).

${ }^{13}$ F. A. Andersen, B. Bak, and S. Brodersen, J. Chem. Phys. 24, 989 (1956).

${ }^{14}$ M. Baba, M. Saitoh, K. Taguma, K. Shinohara, K. Yoshida, Y. Semba, S. Kasahara, N. Nakayama, H. Goto, T. Ishimoto, and U. Nagashima, J. Chem. Phys. 130, 134315 (2009).

${ }^{15}$ M. Oldani and A. Bauder, Chem. Phys. Lett. 108, 7 (1984).

${ }^{16}$ M. Oldani, R. Widmer, G. Grassi, and A. Bauder, J. Mol. Struct. 190, 31 (1988).

${ }^{17}$ T. J. Balle and W. H. Flygare, Rev. Sci. Instrum. 52, 33 (1981).

${ }^{18}$ J.-U. Grabow and W. Stahl, Z. Naturforsch. 51a, 923 (1996).

${ }^{19}$ J. Kraitchman, Am. J. Phys. 21, 17 (1953).

${ }^{20}$ K. Tamagawa, T. Iijima, and M. Kimura, J. Mol. Struct. 30, 243 (1976).

${ }^{21}$ Y. Morino, K. Kuchitsu, and T. Oka, J. Chem. Phys. 36, 1108 (1962).

${ }^{22}$ K. Kuchitsu, J. Chem. Phys. 44, 906 (1966).

${ }^{23}$ C. C. Costain, J. Chem. Phys. 29, 864 (1958).

${ }^{24}$ J. Gauss and J. F. Stanton, J. Phys. Chem. A. 104, 2865 (2000).

${ }^{25}$ T. Oka and Y. Morino, J. Mol. Spectrosc. 6, 472 (1961).

${ }^{26}$ T. Oka, J. Mol. Struct. 352/353, 225 (1995). 